\title{
DECOMPOSABILITY PRESERVING CURVATURE OPERATORS WITH AN APPLICATION TO EINSTEIN MANIFOLDS
}

\author{
MICHAEL R. GABEL AND STANLEY M. ZOLTEK
}

\begin{abstract}
In this paper we examine curvature operators that preserve decomposability. In particular, we prove that if at each point of an Einstein manifold $M$ the sectional curvature operator is nonsingular and preserves decomposability, and the sectional curvature is either nonnegative or nonpositive, then $M$ is a space of nonzero constant curvature.
\end{abstract}

1. Introduction. At each point of a Riemannian manifold its curvature tensor $R$ induces a symmetric linear transformation $R_{x}$ on $\Lambda^{2}$ of the tangent space. The representation of this induced "curvature operator", in the form $L \wedge L$ for some linear map $L$, is directly related to local embeddability in Euclidean space (see $[2,6,8])$.

Generally speaking, a first step has been to find conditions to insure that $R= \pm L \wedge L$. In the nonsingular case, this has been solved by Vilms in dimension greater than 4 (see [7]).

We prove here a similar structure theorem in dimension 4. Specifically we show (see Theorem 3.8) that a nonsingular Bianchi decomposability preserving curvature operator on $\Lambda^{2} V$ with $\operatorname{dim} V=4$ is of the form $\pm L \wedge L$ for some symmetric linear isomorphism $L: V \rightarrow V$.

The techniques used are similar to those of Vilms' in that we both rely on a theorem of Chow [1] on the transformations of the Grassmann variety.

It is well known that Einstein manifolds of dimension 3 are spaces of constant curvature (see [4]). We use our structure theorem in dimension 4 and Vilms' in dimension greater than 4 to give necessary and sufficient conditions for a (connected) Einstein manifold of dimension $\geqslant 4$ to be a space of constant curvature. The condition is that at each point $x$ of the manifold, the induced curvature operator, $R_{x}$, is nonsingular, preserves decomposability and has sectional curvature which is nonnegative or nonpositive.

2. Preliminaries. Let $V$ be an $n$-dimensional real inner product space. By $\Lambda^{p}(V)$, $1 \leqslant p \leqslant n$, we mean the space of $p$-vectors of $V$ together with the naturally induced

Received by the editors September 23, 1982 and, in revised form, May 26, 1983. Presented by S. Zoltek at the winter meeting of the AMS in January 1982 at Cincinnati, Ohio.

1980 Mathematics Subject Classification. Primary 53B20; Secondary 53C25.

Key words and phrases. Curvature operator, decomposability preserving, Einstein manifold, constant curvature. 
inner product from $V$. A 2-vector $\omega \in \Lambda^{2}(V)$ is said to be decomposable if $\omega=x \wedge y$ where $x, y \in V$. A map $\eta: \Lambda^{2}(V) \rightarrow \Lambda^{2}(V)$ preserves decomposability if $\eta(\omega)$ is decomposable for each decomposable 2-vector $\omega$.

The following relationships between elements of $\Lambda^{2}(V)$ and linear subspaces of $V$ are easily verified.

Lemma 2.1. (1) If $x, y \in V, x \neq 0$ and $y \neq 0$, then $x \wedge y \neq 0 \Leftrightarrow$ dimension $\operatorname{span}\{x, y\}=2$.

(2) If $x, y, u, v \in V$, then $x \wedge y \wedge u \wedge v=0 \Leftrightarrow$ dimension $\operatorname{span}\{x, y, u, v\}<4$.

(3) If $x, y, u, v \in V, x \wedge y \neq 0$ and $u \wedge v \neq 0$, then $x \wedge y=c u \wedge v$ for some real number $c \neq 0 \Leftrightarrow \operatorname{span}\{x, y\}=\operatorname{span}\{u, v\}$.

(4) If $x, y, u, v \in V$ with $x \wedge y \neq 0$ and $u \wedge v \neq 0$, then $x \wedge y \wedge u \wedge v=0 \Leftrightarrow$ $\operatorname{span}\{x, y\}$ and $\operatorname{span}\{u, v\}$ contain a common line.

(5) Let $\alpha, \beta \in \Lambda^{2}(V)$ be decomposable. Then $\alpha \wedge \beta=0 \Leftrightarrow \alpha+\beta$ is decomposable.

The Grassmannian $G$ of oriented 2-planes in $V$ will be identified with the set of all decomposable vectors of length one in $\Lambda^{2}(V)$.

Let $V_{n}=\{n$-dimensional subspaces of $V\}$. We say that $P, Q \in V_{2}$ are adjacent if they contain a common line, and that a map $\phi: V_{2} \rightarrow V_{2}$ preserves adjacency if whenever $P$ and $Q$ are adjacent then so are $\phi(P)$ and $\phi(Q)$. If, in adaition, $\phi(P)$ and $\phi(Q)$ being adjacent implies that $P$ and $Q$ are adjacent, we say that $\phi$ preserves adjacency both ways. We say $\phi$ is induced by a linear transformation if there is a linear map $L: V \rightarrow V$ such that $\phi(P)=L(P)$ for all $P \in V_{2}$.

A linear transformation $L: V \rightarrow V$ induces a linear transformation $L \wedge L: \Lambda^{2}(V)$ $\rightarrow \Lambda^{2}(V)$ by setting $L \wedge L(x \wedge y)=L x \wedge L y$ and extending linearly. It is easily checked that this induced map is well defined and that if $L$ is symmetric then so is $L \wedge L$.

A curvature operator $R$ is a symmetric linear operator on $\Lambda^{2}(V)$. Its sectional curvature is the real valued function $\sigma_{R}(P)=\langle R P, P\rangle, P \in G$. We say that $R$ satisfies the Bianchi identity if

$$
\langle R x \wedge y, z \wedge v\rangle+\langle R y \wedge z, x \wedge v\rangle+\langle R z \wedge x, y \wedge v\rangle=0 \quad \forall x, y, z, v \in V .
$$

When $V$ is oriented, has dimension 4 and has orthonormal basis $\left\{e_{1}, \ldots, e_{4}\right\}$ consistent with the given orientation, we can define the Hodge star operator $*: \Lambda^{2}(V) \rightarrow \Lambda^{2}(V)$ by $\langle * \alpha, \beta\rangle=\left\langle\alpha \wedge \beta, e_{1} \wedge \cdots \wedge e_{4}\right\rangle$ where $\alpha, \beta \in \Lambda^{2}(V)$. It is easily checked that this definition is independent of the choice of oriented orthonormal basis for $V$. We note that $*$ does not satisfy the Bianchi identity.

Let dimension $V=4$. We define $\perp: V_{2} \rightarrow V_{2}$ by $\perp(P)=\{v \in V:\langle v, w\rangle=0$ $\forall w \in P\}$ and $\perp^{\prime}: V_{3} \rightarrow V_{1}$ by $\perp^{\prime}(Q)=\{v \in V:\langle v, w\rangle=0 \forall w \in Q\}$.

3. Algebraic results. In this section, for dimension $V=4$, we give necessary and sufficient conditions for a curvature operator to be of the form $\pm L \wedge L$ where $L$ is a nonsingular symmetric linear transformation of $V$ onto itself.

The following theorem is a special case of a result by Wei-Liang Chow [1]. 
THEOREM 3.1. Let $V$ be a 4-dimensional real vector space. If $\phi: V_{2} \rightarrow V_{2}$ is 1-1, onto and preserves adjacency both ways, then either

(1) $\exists \alpha: V_{1} \rightarrow V_{1}$ which is $1-1$, onto and such that $\forall l \in V_{1},\{\phi(P): P \supset l\}=\{P \in$ $\left.V_{2}: P \supset \alpha(l)\right\}$ or

(2) $\exists \beta: V_{1} \rightarrow V_{3}$ which is $1-1$, onto and such that $\forall l \in V_{1},\{\phi(P): P \supset l\}=\left\{P \in V_{2}\right.$ : $P \subset \beta(l)\}$.

Moreover, (1) occurs $\Leftrightarrow \exists$ a nonsingular linear map $L: V \rightarrow V$ such that for all $P \in V_{2}, \phi(P)=L(P)$.

COROLlaRY 3.2. With the assumptions and notation as in the theorem, assume $V$ is endowed with an inner product $\langle$,$\rangle . Then \exists$ a linear isomorphism $L: V \rightarrow V$ such that either $\phi(P)=L(P) \forall P \in V_{2}$ or $(\perp \circ \phi)(P)=L(P) \forall P \in V_{2}$.

Proof. If (1) as in the theorem occurs, then we are done. Assume (2) occurs. Then $\exists \beta: V_{1} \rightarrow V_{3}$ such that $\{\phi(P): P \supset l\}=\left\{P \in V_{2}: P \subset \beta(l)\right\}$. Consider $\psi=\perp \circ \phi$. It is easily checked that $\psi$ is $1-1$, onto and preserves adjacency both ways. Since (2) holds for $\phi,\{(\perp \circ \phi)(P): P \supset l\}=\{\perp(P): P \subset \beta(l)\}$. But $\{\perp(P): P \subset \beta(l)\}=$ $\left\{P: P \supset\left(\perp^{\prime} \circ \beta\right)(l)\right\}$. Thus (1) holds for $\psi$ with $\alpha=\perp^{\prime} \circ \beta$.

Given a linear isomorphism $R: \Lambda^{2}(V) \rightarrow \Lambda^{2}(V)$ which preserves decomposability, define $[R]: V_{2} \rightarrow V_{2}$ by $[R](\operatorname{span}\{x, y\})=\operatorname{span}\{u, v\}$ where $R(x \wedge y)=\mu \wedge v$. That this map is well defined follows from Lemma 2.1 .

Proposition 3.3. If $R: \Lambda^{2}(V) \rightarrow \Lambda^{2}(V)$ is a linear isomorphism which preserves decomposability, then:

(1) $[R]$ is $1-1$.

(2) $[R]$ preserves adjacency.

(3) If either (a) $R(\alpha)$ decomposable $\Rightarrow \alpha$ decomposable or (b) $\forall$ decomposable $\alpha, \beta \in \Lambda^{2}(V), R \alpha \wedge R \beta=0 \Rightarrow \alpha \wedge \beta=0$ or (c) $R$ is symmetric, then $[R]$ is onto and preserves adjacency both ways.

Proof. Part (1) follows from Lemma 2.1.

To prove (2) suppose that $P$ and $Q$ are adjacent. Then $P=\operatorname{span}\{m, l\}$ and $Q=\operatorname{span}\{n, l\}$ for some $m, n, l \in V$. By Lemma 2.1(4), since $R$ preserves decomposability we need only show that $R(m \wedge l) \wedge R(n \wedge l)=0$. But $R(m \wedge l+n \wedge l)$ is decomposable. So $R(m \wedge l+n \wedge l) \wedge R(m \wedge l+n \wedge l)=0$. But then

$$
2 R(m \wedge l) \wedge R(n \wedge l)=0
$$

and (2) follows.

Now we will prove (3). That hypotheses (a) and (b) are equivalent follows from Lemma 2.1(5). In [8], Vilms shows explicitly that hypothesis (c) implies hypothesis (b). Thus we need only show (3) follows under hypothesis (a). Under (a) it is easy to check that $[R]$ is onto. So, let $P=\operatorname{span}\{x, y\}$ and $Q=\operatorname{span}\{u, v\}$ be such that $[R](P)$ and $[R](Q)$ are adjacent. We must show that $P$ and $Q$ are adjacent.

Let $l$ be a vector common to $[R](P)$ and $[R](Q)$. Then

$$
\begin{aligned}
R(x \wedge y+u \wedge v) & =R(x \wedge y)+R(u \wedge v)=m \wedge l+n \wedge l \\
& =(m+n) \wedge l \text { for some } m, n \in V .
\end{aligned}
$$


Thus $R(x \wedge y+u \wedge v)$ is decomposable and hence, by assumption, so is $x \wedge y+u \wedge v$.

So

$$
0=(x \wedge y+u \wedge v) \wedge(x \wedge y+u \wedge v)=2(x \wedge y \wedge u \wedge v)
$$

But then $P$ and $Q$ are adjacent by Lemma 2.1(4).

Proposition 3.4 (VILms [8]). Let $R: \Lambda^{2}(V) \rightarrow \Lambda^{2}(V)$ be a linear isomorphism which preserves decomposability. Assume $[R]$ is induced by a linear map. Then $R= \pm L \wedge L$ for some linear transformation $L: V \rightarrow V$.

LEMMA 3.5. Let $V$ be a 4-dimensional real inner product space and $*: \Lambda^{2}(V) \rightarrow \Lambda^{2}(V)$ and $\perp: V_{2} \rightarrow V_{2}$ be defined as in $\S 2$. Then $[*]=\perp$.

Proof. Let $P \in V_{2}$ and $\left\{u_{1}, \ldots, u_{4}\right\}$ be an orthonormal basis for $V$ such that $P=\operatorname{span}\left\{u_{1}, u_{2}\right\}$. Checking that $*\left(u_{1} \wedge u_{2}\right)=c u_{3} \wedge u_{4}$ for some real number $c$ and applying Lemma $2.1(3)$ completes the proof.

THEOREM 3.6. Let $V$ be a 4-dimensional real inner product space and $R: \Lambda^{2}(V) \rightarrow$ $\Lambda^{2}(V)$ a linear isomorphism. Assume $R$ preserves decomposability and that $[R]$ is onto and preserves adjacency both ways. Then there exists a linear isomorphism $L: V \rightarrow V$ such that either $R= \pm L \wedge L$ or $R= \pm * L \wedge L$.

Proof. Corollary 3.2 applies to $[R]$ and so there exists a linear isomorphism $M$ : $V \rightarrow V$ such that either $[R](P)=M(P) \forall P \in V_{2}$, or $(\perp \circ[R])(P)=M(P) \forall P \in$ $V_{2}$. By Proposition 3.4 and Lemma $3.5 R= \pm L \wedge L$ or $* R= \pm L \wedge L$ for some linear map $L: V \rightarrow V$. Noting that $* 2=$ identity completes the proof.

Proposition 3.7 (Vilms [7]). Let dimension $V \geqslant 3$. Assume that $R: \Lambda^{2}(V) \rightarrow \Lambda^{2}(V)$ is a nonsingular curvature operator satisfying the Bianchi identity. If $R= \pm L \wedge L$, then $L$ is symmetric.

THEOREM 3.8. Let $V$ be a 4-dimensional real inner product space. A curvature operator $R$ is

(1) nonsingular,

(2) satisfies the Bianchi identity,

(3) preserves decomposability

if and only if there exists a symmetric linear isomorphism $L: V \rightarrow V$ such that $R= \pm L \wedge L$.

Proof. By Theorem 3.6, $R= \pm L \wedge L$ or $R= \pm * L \wedge L$. We will show that $R= \pm * L \wedge L$ contradicts assumption (2). So, assume $R= \pm * L \wedge L$. Then

$$
\begin{aligned}
\langle * L \wedge L(x \wedge y), z \wedge v\rangle & +\langle * L \wedge L(y \wedge z), x \wedge v\rangle \\
& +\langle * L \wedge L(z \wedge x), y \wedge v\rangle=0 \quad \forall x, y, z, v \in V .
\end{aligned}
$$

Equivalently, with $\delta$ a generator of $\Lambda^{4}(V)$,

$$
\begin{aligned}
\langle L x \wedge L y \wedge z \wedge v, \delta\rangle & +\langle L y \wedge L z \wedge x \wedge v, \delta\rangle \\
& +\langle L z \wedge L x \wedge y \wedge v, \delta\rangle=0 \quad \forall x, y, z, v \in V .
\end{aligned}
$$


Setting $L x=v$ gives $\langle L y \wedge L z \wedge x \wedge L x, \delta\rangle=0 \forall x, y, z \in V$. Thus,

$$
L y \wedge L z \wedge x \wedge L x=0 \quad \forall x, y, z \in V .
$$

It follows by Lemma 2.1(2) that $\{x, L x, L y, L z\}$ is a dependent set of vectors.

We next show that for each $x \in V, L x=C_{x} x$ for some real number $C_{x}$. Fix $x$ and assume $x$ and $L x$ are independent. So, $\{x, L x\}$ can be extended to a basis $\{x, L x, u, v\}$ for $V$. Since $L$ is nonsingular there exist $y, z \in V$ such that $L y=u$ and $L z=V$. But then $\{x, L x, u, v\}=\{x, L x, L y, L z\}$ is a dependent set of vectors, which is a contradiction.

So, indeed, $\forall x \in V, L x=C_{x} x$ for some real number $C_{x}$.

We now show $C_{x}$ does not depend on $x$. Assume $x$ and $y$ are independent. By the linearity of $L, C_{x} x+C_{y} y=C_{x+y} x+C_{x+y} y$. Since $x$ and $y$ are independent we have that $C_{x}=C_{x+y}=C_{y}$. But then $R= \pm k^{*}$ for some real numbers $k \neq 0$. This contradicts assumption (2). That the converse holds is easily checked.

REMARK. The above result for $\operatorname{dim} V>4$ appears in [7].

4. Geometric applications. In this section we give necessary and sufficient conditions for an $n$-dimensional Einstein manifold, $n \geqslant 4$, to be a space of constant curvature $k, k \neq 0$.

Given a curvature operator $R: \Lambda^{2}(V) \rightarrow \Lambda^{2}(V)$, its Ricci contraction $r(R)$ is the symmetric linear transformation on $V$ given by

$$
\langle r(R)(v), w\rangle=\sum_{i}\left\langle R\left(v \wedge e_{i}\right), w \wedge e_{i}\right\rangle
$$

where $\left\{e_{1}, \ldots, e_{n}\right\}$ is an orthonormal basis for $V$. It is easily checked that this definition is basis free.

A Riemannian manifold is called an Einstein manifold if its Ricci tensor is a constant multiple of the metric tensor. At a point this condition implies that $r(R)=k I$ for some real number $k$.

THEOREM 4.1. Let $M$ be an $n$-dimensional (connected) Einstein manifold, $n \geqslant 4$, and let $R$ be the induced curvature operator at some point of $M$. Assume $R$ is nonsingular and preserves decomposability. Then

(a) $\sigma_{R} \geqslant 0 \Rightarrow R=k I \wedge I$ with $k>0$,

(b) $\sigma_{R} \leqslant 0 \Rightarrow R=k I \wedge I$ with $k<0$.

Proof. By Theorem 3.8, $R= \pm L \wedge L$ where $L$ is symmetric and nonsingular. So there exists an orthonormal basis $\left\{e_{1}, \ldots, e_{n}\right\}$ for $V$ such that $L e_{i}=a_{i} e_{i}, a_{i} \neq 0$. Then $\left\{e_{i} \wedge e_{j}: i<j\right\}$ is an orthonormal basis for $\Lambda^{2}(V)$ such that $R e_{i} \wedge e_{j}=a_{i} a_{j} e_{i}$ $\wedge e_{j} \forall i, j$ or $R e_{i} \wedge e_{j}=-a_{i} a_{j} e_{i} \wedge e_{j} \forall i, j$. It suffices to show that $a_{k}=a_{l} \forall k, l$. Since $r(R)$ is a multiple of the identity, it follows that, for some constant, $c$, $\left\langle r(R) e_{j}, e_{j}\right\rangle=c$ for each $j$. Equivalently, $c=\sum_{i \neq j} a_{j} a_{i}$ for each $j$. Now, let $k \neq l$. Then $\sum_{i \neq k} a_{k} a_{i}=\sum_{i \neq l} a_{l} a_{i}$. Set $S(k, l)=a_{1}+\cdots+\hat{a}_{k}+\cdots+\hat{a}_{l}+\cdots+a_{n}$. Thus $a_{k} S(k, l)=a_{l} S(k, l)$.

We will be done if we show $S(k, l) \neq 0$. This will certainly be true if we show that $a_{1}, \ldots, a_{n}$ have the same sign. 
First assume $\sigma_{R} \geqslant 0$. We know that $R= \pm L \wedge L$. We claim $R=+L \wedge L$, for if $R=-L \wedge L$ then $0 \leqslant \sigma_{R}\left(e_{i} \wedge e_{j}\right)=-a_{i} a_{j} \forall i$, j. Since $L$ is nonsingular, we have $a_{i} \neq 0 \forall i$, so that, in fact, $0<-a_{i} a_{j} \forall i, j$. But this is impossible as $n \geqslant 3$. So $R=+L \wedge L$. Since $\sigma_{R} \geqslant 0$ and $L$ is nonsingular, we now have $0<a_{i} a_{j} \forall i, j$ and thus, when $\sigma_{R} \geqslant 0, a_{1}, \ldots, a_{n}$ are of the same sign.

Now assume $\sigma_{R} \leqslant 0$. An argument similar to the one above gives that $R=-L \wedge L$, which together with the nonsingular of $L$ implies that $0>-a_{i} a_{j} \forall i, j$. And so, when $\sigma_{R} \leqslant 0, a_{1}, \ldots, a_{n}$ are of the same sign.

Thus, in either case, $a_{1}, \ldots, a_{n}$ have the same sign, so the theorem follows.

COROllary 4.2. Let $M$ be an $n$-dimensional Einstein manifold with $n \geqslant 4$. Then the following two statements are equivalent.

(A) $M$ is a space of nonzero constant curvature.

(B) At each point the curvature operator is nonsingular, preserves decomposability and has sectional curvature which is either nonnegative or nonpositive.

Proof. That (A) implies (B) is clear. We now show that (B) implies (A).

For each $x \in M$, let $R_{x}$ denote the sectional curvature at $x$. Then, by Theorem 4.1, $R_{x}=k_{x} I \wedge I$ for some nonzero constant $k_{x}$. So $\sigma_{R_{x}}(P)$, for $P \in G$, depends only on $x$ and not on $P$. So by a theorem of Schur $[3,5], M$ is a space of nonzero constant curvature.

\section{REFERENCES}

1. W.-L. Chow, On the geometry of algebraic homogeneous spaces, Ann. of Math. (2) 50 (1949), 32-67.

2. H. Jacobowitz, Curvature operators on the exterior algebra, Linear and Multilinear Algebra 7 (1979), 93-105.

3. S. Kobayashi and K. Nomizu, Foundations of differential geometry, Vol. I, Interscience, New Yor . 1963.

4. J. A. Schouten and D. J. Struik, On some properties of general manifolds relating to Einstein's theory of gravitation, Amer. J. Math. 43 (1921), 213-216.

5. F. Schur, Über den Zusammenhang der Raüme konstanter Krümmungsmasses mit den projectiven Raümen, Math. Ann. 27 (1880), 537-567.

6. T. Y. Thomas, Riemannian spaces of class one and their characterizations, Acta Math. 67 (1936), 169-211.

7. J. Vilms, Factorization of curvature operators, Trans. Amer. Math. Soc. 260 (1980), 595-605.

8. L_L Local isometric imbedding of Riemannian n-manifolds into Euclidean $(n+1)$-space, J. Differential Geom. 12 (1977), 197-202.

Department of Mathematical Sciences, George Mason University, Fairfax, Virginia 22030 